\title{
HELIOS: A MULTI-PURPOSE LIDAR SIMULATION FRAMEWORK FOR RESEARCH, PLANNING AND TRAINING OF LASER SCANNING OPERATIONS WITH AIRBORNE, GROUND-BASED MOBILE AND STATIONARY PLATFORMS
}

\author{
S. Bechtold ${ }^{\mathrm{a}}$, B. Höfle $\mathrm{a}^{\mathrm{*}}$ \\ ${ }^{a}$ Institute of Geography, GIScience, LiDAR Research Group (LRG), Heidelberg University, Heidelberg, Germany \\ - helios@ sebastian-bechtold.de, hoefle@uni-heidelberg.de
}

Commission III, WG III/2

KEY WORDS: HELIOS, Laser Scanning, LiDAR, Simulation, Survey Planning, Virtual Reality, Java, XML

\begin{abstract}
:
In many technical domains of modern society, there is a growing demand for fast, precise and automatic acquisition of digital 3D models of a wide variety of physical objects and environments. Laser scanning is a popular and widely used technology to cover this demand, but it is also expensive and complex to use to its full potential. However, there might exist scenarios where the operation of a real laser scanner could be replaced by a computer simulation, in order to save time and costs. This includes scenarios like teaching and training of laser scanning, development of new scanner hardware and scanning methods, or generation of artificial scan data sets to support the development of point cloud processing and analysis algorithms. To test the feasibility of this idea, we have developed a highly flexible laser scanning simulation framework named Heidelberg LiDAR Operations Simulator (HELIOS). HELIOS is implemented as a Java library and split up into a core component and multiple extension modules. Extensible Markup Language (XML) is used to define scanner, platform and scene models and to configure the behaviour of modules. Modules were developed and implemented for (1) loading of simulation assets and configuration (i.e. 3D scene models, scanner definitions, survey descriptions etc.), (2) playback of XML survey descriptions, (3) TLS survey planning (i.e. automatic computation of recommended scanning positions) and (4) interactive real-time 3D visualization of simulated surveys. As a proof of concept, we show the results of two experiments: First, a survey planning test in a scene that was specifically created to evaluate the quality of the survey planning algorithm. Second, a simulated TLS scan of a crop field in a precision farming scenario. The results show that HELIOS fulfills its design goals.
\end{abstract}

\section{INTRODUCTION}

Laser scanning is a widely used method of acquiring high-detail, high-precision 3D models of physical objects in many technical fields of modern society like construction, mining, farming and forestry, or science. The technology is advancing quickly, and increasingly capable laser scanners are introduced every year. A remaining problem is that laser scanners are still very expensive, and it is unlikely that they will become significantly more affordable in the foreseeable future. However, there are use cases where it might be possible to replace the operation of a real laser scanner with a computer simulation, resulting in a massive reduction of costs and effort. If the focus of interest in a laser scanning operation is on the acquisition of some very specific real-world data, it can never be replaced by a simulation. However, there are many questions in laser scanning research for which the answers lie not in the actual content of the captured data, but in its structural characteristics, and these can very well be reproduced in a simulated environment. Possible use cases for a laser scanning simulator are, e.g., research and planning of scanning strategies, laser scanning teaching and training, generation of artificial scan data for algorithm development, or sensor development and evaluation. More detailed thoughts on the usefulness of a laser scanning simulator were presented by Lohani and Mishra (2007).

\section{RELATED WORK}

The general idea to simulate laser scanning is not new. A significant increase in publications on the topic can be observed since around 2005. Most publications focus on methods and algorithms

${ }^{*}$ Corresponding author to achieve high simulation realism for one very specific aspect of the light detection and ranging (LiDAR) technology, or for very specific applications like forestry (e.g. Lovell et al. (2005)). The following paragraphs provide an overview of some selected publications with an emphasis on their unique characteristics.

Lohani and Mishra (2007) have developed an airborne laser scanning (ALS) simulator for education and general research. The application features a user-friendly graphical user interface (GUI) which includes a scene generator/editor and a graphical flight path planner, turning the software into a complete ready-to-use ALS simulation suite. However, the LiDAR simulation method described by Lohani and Mishra (2007) works with "2.5D" elevation maps only. Overhanging geometry is not supported, the scene is meant to be scanned exclusively from above. Therefore, the presented approach cannot be used to simulate terrestrial laser scanning (TLS) with realistic high-detail scenes.

A critical aspect of TLS simulation is the support of scene data structures that allow detailed representation of objects with complex geometry (e.g. vegetation), suited for realistic simulation of LiDAR measurements from close distances and from any direction (as opposed to $2.5 \mathrm{D}$ elevation maps, which only allow meaningful measurements from above). A project that takes this into account is presented by Kim et al. (2009). In contrast to the system described by Lohani and Mishra (2007), they use true $3 \mathrm{D}$ geometry to describe the scanned scene. This approach may make their software potentially usable for meaningful TLS simulation as well. However, this is not discussed in the paper.

One solution which was explicitly designed for TLS simulation was published by Wang et al. (2013). They have modeled the interaction between a RIEGL VZ-1000 TLS system and a Tilia 
tree, represented by a triangle mesh. The purpose of their implementation is the investigation of the effects of different scanning positions (distance between tree and scanner, and elevation of the scanner above ground) on the derivation of plant characteristics like leaf area index from the generated point cloud. The publication does not mention to which extent their software could be used for other purposes, or how easily it could be modified and extended.

An important aspect to point out here is that none of the solutions mentioned above simulate beam divergence - the fact that a laser beam is not an infinitely thin line, but a cone of light which intersects with surfaces in an elliptical area, called the laser's footprint. This has a number of implications on how the light is reflected by surfaces and detected by the LiDAR sensor. Modern laser scanners take these effects into account and measure the socalled full waveform of a reflected laser pulse. In contrast to the single point coordinates which are returned by less sophisticated scanner models, the full waveform contains valuable additional information which can be used by LiDAR researchers to learn more about the nature of the scanned objects. For example, this is especially useful for LiDAR-based studies of vegetation, due to the complex geometry of plants (e.g. Hollaus et al. (2009), Höfle et al. (2012)).

Kukko and Hyyppä (2009) have developed a laser scanning simulator which is capable of modeling beam divergence and fullwaveform signal recording. Generally, their model of the physics involved in LiDAR measurements is very detailed, resulting in a high level of simulation realism. However, just like the solution presented by Lohani and Mishra (2007), their simulator uses 2.5D elevation maps to represent scene geometry, so that it can only be used for ALS simulation.

A TLS simulation that includes modeling of beam divergence and full-waveform signal detected is described by Hodge (2010). Unusual for a TLS simulation, this implementation uses 2.5D elevation maps, which is explained by the fact that it was specifically designed to analyze errors in high-resolution TLS scanning of uneven surfaces (e.g. pebble-covered ground) from close distances (ca. $3 \mathrm{~m}$ ). The system is designed to work with scene models at scales below ca. $1 \times 1 \mathrm{~m}$ and a single scanning position. Scanner platform movement is not supported. However, the implementation models radiometric as well as geometric aspects of highresolution, small-distance laser scanning with high realism.

\section{OUR APPROACH}

Laser scanning is a highly versatile technology with many different fields of application, and several different modes of operation (e.g. airborne, stationary terrestrial, mobile terrestrial, and even naval). Accordingly, there are just as many possible applications for a laser scanning simulator. A study of existing literature has shown that most previous research projects on laser scanning simulation were focusing on highly detailed modeling of small parts of this wide field, like one specific mode of operation (e.g. ALS), or one specific aspect of the complex physics of LiDAR measurements.

We decided to follow an opposite approach and started to develop a flexible multi-purpose simulation framework which could support many different types of laser scanning research projects. More specifically, the system is designed to have the following characteristics:

- Support for many different types of environments (scan targets), scanners, platforms and survey methods, ground-based as well as airborne, stationary as well as mobile.
- Especially, support for scenes with true 3D geometry (as opposed to " $2.5 \mathrm{D}$ "). This is an essential requirement for realistic TLS simulation.

- A "data-driven" simulation engine: As many aspects as possible should be stored in configuration files, so that they can be changed easily.

- Support for easy modification and extension, to provide a solid foundation for many different aspects of laser scanning research and allow "rapid prototyping" of new features and experiments.

- Based on free, open-source and platform-independent software, to enable and encourage a large group of potential users to try and possibly modify and extend the program to meet their requirements.

We created a modular and highly configurable laser scanning simulation framework called HELIOS, that incorporates all of these ideas. As already mentioned, its development follows a "breadth first" strategy: We decided to focus on simple implementations of many aspects first, instead of starting with complex implementations of few aspects. This leads to the situation that some features are not yet developed as far as one might expect of a mature, full-featured LiDAR simulator, while others are new and unique - especially combined in a single software package. Also, some features are already stable and well-tested, while others still have an experimental character.

\subsection{Software Architecture}

HELIOS is written in the Java programming language, which supports fast development, high runtime performance and very good multi-platform support, so that the software can run out-ofthe-box on Windows, Linux and Mac OS operating systems.

The system is designed as a modular library that can be used to develop different types of applications that make use of its laser scanning simulation capabilities. In theory, this includes desktop applications as well as web-based solutions (e.g. through inclusion of HELIOS into a Java Web Servlet) and even mobile apps. However, only PC desktop applications have been implemented and tested so far.

The HELIOS library consists of a core package and a number of modules that perform different tasks. The core package contains classes that model the three main components of the simulation: The platform (Section 4.), the scene (Section 5.) and the scanner (Section 6.). Each simulation that is run with HELIOS represents a series of interactions between these three components.

Modules are built on top of the core components and provide extended functionality for different tasks. Currently, there are modules for the following tasks:

- Loading of simulation assets (i.e. scene, platform and scanner definitions) from files

- Playback of pre-defined surveys

- Real-time 3D visualization

- Survey planning 
Applications are developed by including the core and one or more module packages and connecting them in the desired way. Not all modules might be required to implement a specific simulation task, and existing modules could be replaced with others that perform the same tasks in a different way. The following sections take a closer look at the different modules and components of the system, and describe their current capabilities and how they work together.

\section{THE PLATFORM (CORE COMPONENT)}

The platform component simulates the platform on which the scanner is mounted. All changes of the scanner's position and orientation in space are caused and controlled by the platform component. HELIOS implements multiple platform classes that can be used to simulate different types of aircraft and ground vehicles, as well as stationary platforms.

An actual implementation of a specific platform consists of two parts: A class which contains the logic to simulate the general platform type (e.g. a wheeled ground vehicle or a helicopter) and a set of parameters that define the specific characteristic of the platform (e.g. mass, maximum speed, position and attitude of the scanner mount, etc.)

Currently, HELIOS implements the following platform classes:

- Four-wheel ground vehicle with one steerable axle

- Helicopter/Multicopter

- Simple linearly interpolated movement along straight lines

- "Dummy" platform without movement code (base class of all other platform classes, can be used to simulate stationary scanners)

The ground vehicle and helicopter classes use simple physical models with parameters like mass, gravitation and engine forces, to approximate the real-world behaviour of these vehicle types. The linear path movement platform simply interpolates the position of the platform on a straight line between two waypoints with constant speed. The "dummy" platform does not contain any movement code at all. It can be used to simulate stationary platforms like tripods.

As already mentioned above, each platform class has various configuration parameters that can be adjusted to represent specific platform models. These configuration settings can be loaded into the software using the assets loader module (Section 7.).

It is important to note that the platform component does not contain any logic to define trajectories and move along them. Platform classes only control how a platform moves, but not where it moves. In order to actually move a platform through the scene, an additional steering module is required. This role is fulfilled by the survey playback module, described in Section 8 .

\section{THE SCENE (CORE COMPONENT)}

The scene component represents the environment that is scanned by the simulated laser scanner. The scene might also influence the platform's position and/or movement: Stationary scanning positions as well as the trajectory of a moving scan platform need to be planned with the geometry of the scan target in mind. Also, slopes and path-blocking features like walls or vegetation would limit the freedom of movement of ground vehicle platforms and very low flying airborne platforms in the real world. However, no collision detection between platform and scene is implemented in the current version.

From a software engineering point of view, the scene component serves two purposes: First, it contains a data structure that holds scene geometry and materials data. In HELIOS, a scene is defined by a triangle mesh with each triangle holding a reference to a material definition that defines the physical properties of the patch of surface that is represented by the triangle. Material properties affect the strength of laser pulse reflections on a surface.

The second purpose of the scene component is to provide methods for fast intersection tests between a ray, defined by origin and direction $3 \mathrm{D}$ vectors, and the scene geometry. This type of spatial query is called ray casting. HELIOS uses ray casting to simulate the transmission of a laser pulse from the laser scanner to the scene's surface (see Section 6.1 for details).

Modern laser scanners operate with up to a million pulses per second. In order to simulate such devices with optimal speed (ideally, in real-time or faster), a fast raycasting algorithm is mandatory. The ray casting algorithm implemented in HELIOS is based on traversal of a so-called k-dimensional tree (kd-tree), as described by Bentley (1975). A kd-tree is a binary tree data structure that recursively splits the Euclidean 3D space of the scene into smaller sections until each section contains only a small number of geometric primitives (typically something between one and up to a few dozen). During the subdivision process, the positions of the split planes as well as the parent-child-relationships between a split section as a whole and the two smaller regions created by the split are stored in memory. This information can then be used to implement very efficient spatial search algorithms, including ray casting. A description of how exactly HELIOS uses ray casting to simulate laser pulses is given in Section 6 .

\section{THE SCANNER (CORE COMPONENT)}

The scanner component simulates the actual laser scanner. This responsibility consists of three tasks: Simulation of the beam deflection unit, simulation of the firing of laser pulses, and detection and processing of their reflections. The interaction of laser pulses with scene surfaces is modeled in the scene component, instead.

The beam deflection unit is the part of the laser scanner that controls the pattern of angular movement in which the laser beam scans the environment. HELIOS implements different types of beam deflection units and scan patterns (Fig. 1):

- Rotating mirror (parallel scan lines)

- Fiber array (parallel scan lines)

- Oscillating mirror (“zig-zag” scan pattern)

- Conic mirror (elliptical scan pattern, a.k.a. "Palmer scan")

\subsection{Beam Divergence and Full-Waveform Recording}

HELIOS simulates laser beam divergence by approximating the beam's light cone using multiple raycasting queries (Fig. 2). The rays are arranged with evenly distributed angular distances in concentric circles around the central axis of the beam. Subsampling quality can be controlled by setting the number of circles, 


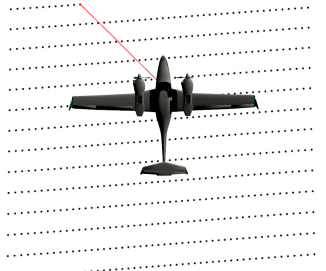

(a)

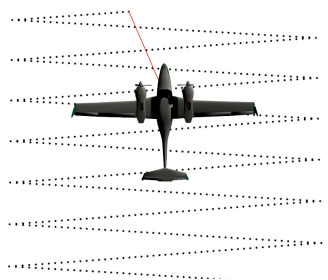

(c)

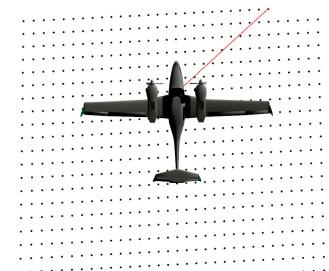

(b)

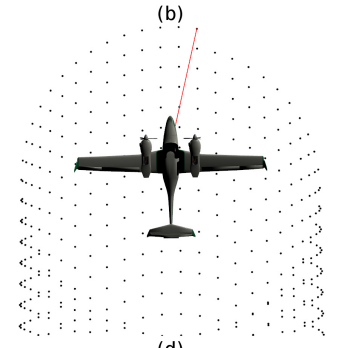

(d)
Figure 1: Different scan patterns supported by HELIOS: (a) rotating mirror, (b) fiber array, (c) oscillating mirror, (d) conic mirror. Aircraft 3D model: CC-BY-SA Emmanuel Baranger

and thus, indirectly, the number of subsampling rays and their density.

For each subsampling ray, a raycasting query is performed on the scene's geometry. If the ray intersects with a primitive, the surface incidence angle and a reference to the material definition of the intersected primitive is returned. This information is used to simulate the interaction of the transmitted waveform with the surface and to compute a reflection waveform for each subsampling ray, taking into account the following additional parameters: energy of the emitted pulse, distance from the emitter, distance from the center of the beam, scanner efficiency, atmospheric attenuation and the BRDF surface reflectance model.

The final recorded full waveform of the entire pulse is created by discretizing and summing up the individual waveforms of each subsampling ray. The temporal resolution of the waveform can be configured by the user. Currently, we are running most of our tests with 500 discretization "bins", which means that each recorded waveform is represented as an array of 500 amplitude values.

Point cloud coordinates are derived by applying echo detection algorithms (Mallet and Bretar (2009)). So far, peak detection and Gaussian decomposition are implemented and can be selected by the user. The point coordinates and the full waveform data for each pulse are then written to separate files. Each point is stored together with a reference to the full waveform data from which it is derived.

\section{THE ASSETS LOADER MODULE}

Before a simulation can be run, data sets that define a scanner, a platform and a scene - so-called simulation assets - need to be loaded. Theoretically, these data sets can come from different sources: It could be entered by the user at runtime, it could be generated procedurally (e.g. a random elevation map), or it could be loaded from files that hold the data in one of many possible formats. Due to this variety, no functionality to load simulation assets data is included in the core. Instead, the required logic is expected to be provided by a module that could be replaced with different implementations, depending on the use case. Currently, HELIOS features an assets loader module that reads asset definitions from XML documents and some additional files (e.g. 3D model files that hold scene geometry data).

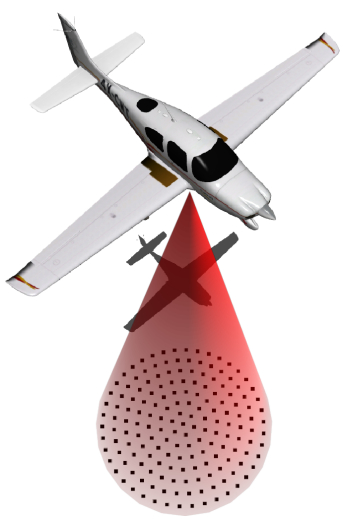

Figure 2: HELIOS simulates beam divergence using multiple subsampling rays. Each black dot represents one subsampling ray. For visualization purposes, the rays themselves are not shown and the illustration is not to scale. Aircraft 3D model: CC-BY-SA Emmanuel Barranger

\subsection{Loading Scene Assets}

The XML definition of a scene does not hold actual geometry data. Instead, geometry data is loaded from separate files in traditional 3D data formats. Currently, HELIOS supports loading of scene geometry data from the Wavefront object 3D model data file format (.OBJ), from the Geographic Tagged Image File Format (GeoTIFF) raster map data format (“2.5D” elevation map), and from "xyz" ASCII point cloud files.

The XML scene description defines how a scene is composed of one or multiple geometry parts that are loaded from different 3D data files, and how the raw data that is read from the files should be preprocessed to build the final scene. Scene part loading and preprocessing is performed by a sequence of one or more so-called filters which are applied to loaded geometry data as defined in the scene description. Currently, the following filters are available:

- Wavefront Object Mesh Loader: This loader reads a triangle mesh with associated material definitions from a Wavefront object (.OBJ) file. This is the standard way of loading a triangle-mesh-based scene geometry into HELIOS.

- GeoTIFF Loader: This loader reads a terrain elevation map from a GeoTIFF file and converts it into a triangle mesh.

- XYZ Point Cloud Loader: This loader reads an ASCII "xyz" point cloud file. The loader subdivides space into a grid of cubic cells (also called "voxels") and checks whether a cell contains at least one point of the point cloud. If this is the case, the cell is defined as "solid", and an axis-aligned bounding box (AABB) primitive with the extent of the cell is created. The voxel method is a simple way of converting point cloud geometry into a solid volume with a surface that can be virtually scanned.

- Coordinate Transformation Filter: This filter can be used to transform scene geometry from one spatial reference system to another. In order to combine multiple scene parts with different coordinate systems into a single scene, all parts need to be transformed to the same coordinate system. It can also be used in the case that only a single scene part is used, but the simulation should for some reason use a different coordinate system than the one in which the part is defined. 


\subsection{Loading Platform and Scanner Assets}

Platforms and scanners are defined through XML alone. No additional resource files are used. However, it is possible to define 3D model files that should be used by the (optional) visualization module to draw the platform and the scanner.

\section{THE SURVEY PLAYBACK MODULE}

An important part of any laser scanning survey is to make and carry out a plan that defines where the scanner should be placed (or, in the mobile case, along which trajectory the platform should move) and which scanner settings should be used for which part of the survey. Following the modular design principles of the HELIOS architecture, the simulator's core does not contain any logic to support these things. Instead, HELIOS features a module that provides this functionality. It reads a survey definition from an XML document and performs an automatic "playback" of the survey.

The survey playback module can be used to define both surveys with a stationary terrestrial scanner (with one or multiple scanning positions) as well as surveys with a moving scanner on a mobile or airborne platform. A survey definition can consist of multiple scans. For each scan, the used platform, scanner and, in the case of mobile/airborne surveys, a sequence of waypoints that describe the platform's path of movement during the scan, can be defined.

\section{THE VISUALIZATION MODULE}

The visualization module provides interactive real-time $3 \mathrm{D}$ visualization of a running simulation. It is based on the JMonkey Engine (JME) library. JME contains functionality like 3D graphics output, user input, physics simulation and networking for computer games and simulations. Currently, the visualization module allows only limited interaction: The user can move the camera (i.e. the position and direction of the 3D view) around the scene and control simulation speed. However, even with only these features, the visualization module is a very useful tool. For example, it can be used to check if a survey playback definition is set up and working correctly, to find programming errors in the simulation code, or to create still images and videos of a simulated survey. Figure 3 shows four different simulated surveys rendered with the visualization module.

\section{THE SURVEY PLANNING MODULE}

In TLS surveys, there is an interest in keeping the number of individual scanning positions as low as possible, since each additional scanning position adds to negative effects like

- an additional amount of time required to transport the scanner to the position, deploy, operate and undeploy it.

- an additional amount of electrical energy required for scanner operations. This is especially critical in situations where no spare batteries or recharging opportunities are available.

- increased redundancy in the produced scan data, leading to an unnecessary increase in file sizes and processing time.

- additional risk of scanner damage (e.g. accidental dropping), or even risk of injury for scanner operators in dangerous terrain.

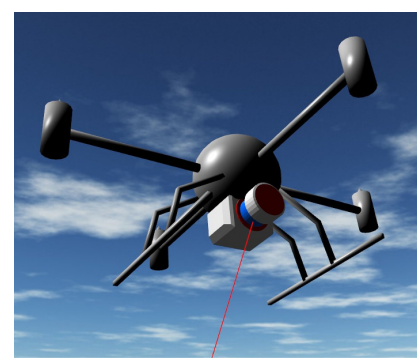

(a)

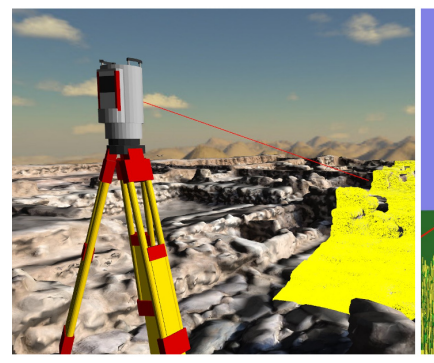

(c)

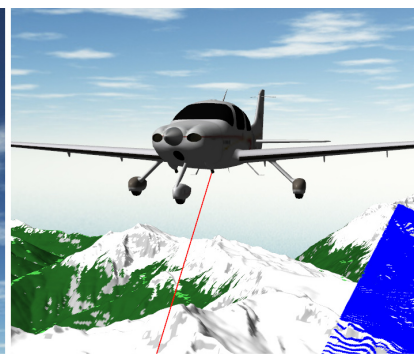

(b)

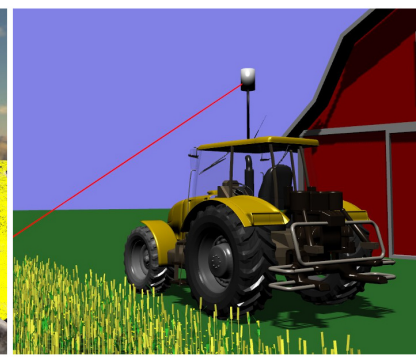

(d)
Figure 3: Surveys with different types of scanner and platforms, rendered by the visualization module: (a) quadrocopter, (b) fixedwing aircraft, (c) tripod, (d) tractor. Aircraft 3D model: CC-BYSA Emmanuel Barranger. Tractor 3D model: tf3dm.com (free for personal use)

However, if the number of scanning positions is too low, parts of the scene may not be captured in the desired resolution, or not at all, leaving "holes" in the data set. Survey planners are challenged to choose scanning positions in such a way that the desired coverage and resolution is achieved, while keeping the total number of scans as low as possible. Especially in environments with very complex geometry, this is not an easy task even for experienced TLS operators.

HELIOS contains an experimental module that provides an approximative solution to the problem of finding the recommended scanning positions (RSPs) for a given scene, based on checking the lines of sight between potential scanning positions (PSPS) and so-called visibility test points (VTPs). A PSP is a place where the scanner could be positioned and operated as part of the survey. The goal of the algorithm is to find out whether or not it is really efficient to put the scanner there. A VTP is a point on the surface of the scene, representing the surrounding surface area to half the distance to the next VTP. By checking the lines of sight between a PSP and each VTP in the scene, an approximation of the PSP's so-called viewshed - the part of the scene that is visible from the PSP - is computed. With knowledge about each PSP's viewshed, decisions can be made about which PSPs should indeed be used in the survey in order to achieve optimal scene coverage, and which PSPs can be dismissed because they would only produce redundant data. The complete algorithm consists of four steps:

1. Define a set of PSPs.

2. Define a set of VTPs.

3. Compute the viewshed of each PSP.

4. Find and remove all PSPs which do not have at least one VTP in their viewshed that is not visible from any other PSP. 


\subsection{Setting up Potential Scanning Positions (PSPs)}

The first observation that should be made when defining PSPs is that the scanner will usually only be placed on horizontal surfaces with little or no slope. It will rarely be positioned on steep slopes, and most probably never on vertical walls.

In order to find PSPs on horizontal surfaces, the survey planning module uses the HELIOS framework's raycasting functionality. In a regular grid over the scene area, with $\mathrm{x} / \mathrm{y}$ step sizes that can be configured by the user, rays are cast vertically "down from the sky", and the first point where the ray intersects with scene geometry (i.e. the highest surface point at the given (x/y) coordinate) is added to the list of PSPs. Pointing straight down, the rays will never hit vertical surfaces (since they run parallel to them), so no points on vertical surfaces will ever be selected. However, the rays will hit slopes. The slope angle at the intersection point is checked, and the point is discarded if the slope exceeds a threshold that can be defined in the XML configuration.

\subsection{Setting up Visibility Test Points (VTPs)}

The set of VTPs is built with the same basic approach as the set of PSPs. Again, raycasting is used to find surface points, but with two major differences:

- Rays are not only cast down vertically from the top, but also horizontally from all four sides of the scene, since (in contrast to PSPs) vertical walls need to be covered with VTPs, too.

- Instead of only selecting the first intersection between a ray and the scene, this time all ray-scene intersections are selected as VTPs, since the entire surface of the scene should be covered.

In most cases, this method of placing VTPs will not result in a regular pattern of evenly distributed VTPs, since most surfaces in the scene are usually not axis-aligned. Especially the complex shapes of natural objects like plants or rocks will produce very irregular VTP distribution patterns. This does not pose a problem as long as the maximum distance between VTPs is small enough to cover the scene with sufficient density.

\subsection{Computing PSP Viewsheds}

Computing the viewshed of a PSP means to check the lines of sight between the PSP and each VTP in the scene. Again, the scene raycasting functionality of the HELIOS core is used: A PSP is defined as the ray's origin, and its direction is defined as the difference vector between the PSP and the VTP. Then, the raycasting query is performed and the algorithm checks where the ray intersects with the scene. If the distance between the first intersection point and the VTP is larger than a predefined threshold $(0.05 \mathrm{~m}$ by default, configurable by the user through XML), no line of sight exists, and the tested VTP is not part of the PSP's viewshed.

\subsection{Finding Recommended Scanning Positions (RSPs)}

The crucial measure for deciding whether or not a PSP is recommended is the number of VTPs that are exclusively visible from this position. In HELIOS terminology, this is called the exclusivity of the PSP. The basic idea of the algorithm, to find a set of RSPs, is to remove all PSPs with exclusivity $=0$, which means that they are fully redundant. It is important to note here that the removal of such a PSP will usually change the exclusivity of some of the remaining PSPs: If a remaining PSP shares at least one of its visible VTPs with the removed PSP, but with no others, the removal will increase this PSP's exclusivity by the number of shared VTPs. Because of this, the order in which redundant PSPs are removed matters.

One straightforward approach is to remove redundant PSPs in ascending order of their viewshed size (i.e. remove the PSP with the smallest viewshed first). The thought behind this is that PSPs with large viewsheds are generally preferable: Since the goal of the overall algorithm is to minimize the number of required scans, the remaining PSPs should cover as much of the scene as possible. However, this approach does not always produce the desired result. In some cases, removing the PSPs with the smallest viewsheds causes the redundant coverage of some VTPs to decrease until they become exclusive for another PSP, which then needs to be kept. This can be avoided by removing PSPs in an alternative order that does not create additional exclusivities.

Experiments have shown that using the arithmetic mean of the line-of-sight incidence angles on the scene surface as the order criterion instead of the viewshed size produces better results for some scenes. The current implementation of the survey planning algorithm supports both methods. The basic algorithm can be summarized as follows:

1. Build the initial sets of VTPs and PSPs and compute each PSP's viewshed.

2. For each PSP, determine exclusivity (i.e. identify and count all VTPs which are only visible from the PSP)

3. If at least one PSP with exclusivity $=0$ exists, remove the one with the smallest viewshed or mean incidence angle (depending on settings) and continue with step 2. Otherwise, the algorithm is finished.

The result is a list of scanning positions that each have at least one exclusive VTP in their viewshed. Altogether, they provide the largest possible coverage of the scene with the minimal number of scan positions.

\subsection{Excluding Dispensable Scanning Positions}

In analogy to inaccessible or unsafe PSPs, which need to be excluded before the RSP search algorithm is run, there may also exist dispensable RSPs, which can only be identified after the search is finished: They have an exclusivity greater than 0 , but the exclusive parts of their viewsheds are so small that the increase in coverage which comes with the addition of these scan positions to the survey is not worth the effort of moving and operating the scanner there. It should be noted that this is difficult to decide algorithmically, since some areas of the scene may be more important than others, and the definition of "importance" that applies here depends very much on things like the nature of the scene, how the acquired data is going to be used, and the individual notions of the people who perform the survey and process the data.

Nevertheless, the algorithm can easily be extended to automatically reduce the number of scan positions based on a simple rule: First, all PSPs with exclusivity $=0$ are removed, just like in the basic version. Then, the PSP with the lowest exclusivity is removed and the exclusivity of all remaining PSPs is re-computed. These steps are repeated until the combined coverage of all remaining PSPs together drops below a specified percentage. In 


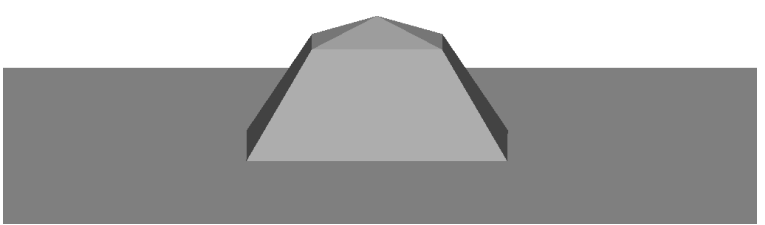

Figure 4: A pyramid scene model used to test the survey planning algorithm.

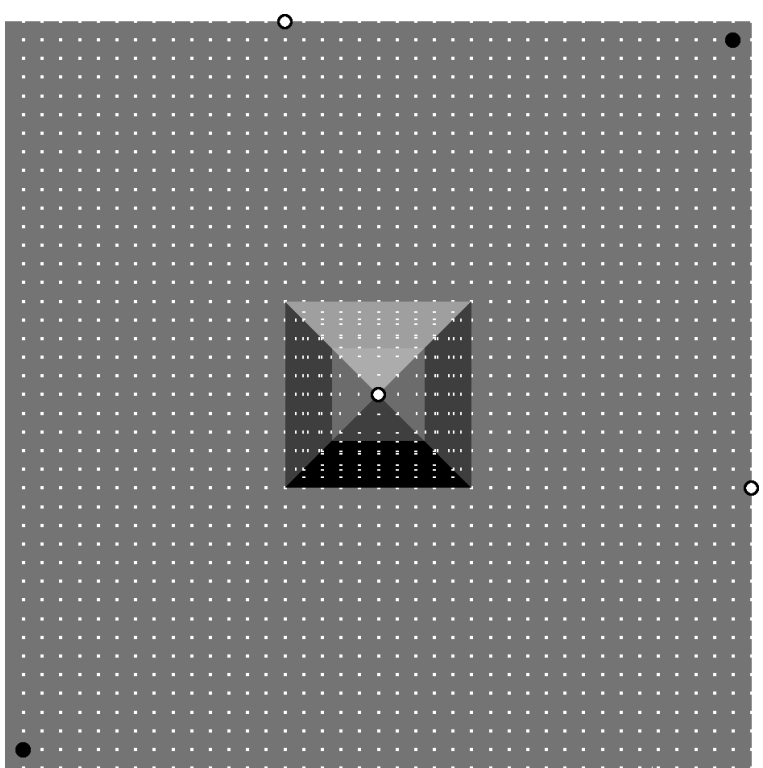

Figure 5: Two different survey planning results for the pyramid scene. Large white points: RSPs with removal order based on viewshed size. Large black points: RSPs with removal order based on mean line-of-sight incidence angle. The small white dots represent PSPs and VTPs simultaneously, since the same grid was used for the placement of both point types.

many scenarios, this method removes quite a large number of additional PSPs. Future research has to investigate to which extent these PSPs match with the ones that would also be discarded by a human decision-maker.

\section{RESULTS}

\subsection{Survey Planning Test: Flattened-top Pyramid Scene}

Artificial scenes with simple, but deliberately designed geometry are helpful tools to verify the correctness of the simulator. One such scene was constructed to test the behaviour of the TLS survey planning module in a special situation where multiple PSPs together cover the (larger) viewshed of another single PSP, and also additional regions exclusively. If this was not the case (i.e. if the single PSP with the large viewshed would cover the viewsheds of the other PSPs completely), the other PSPs could be removed. However, since this is not the case, the single PSP with the large viewshed can be removed instead.

One possible scene in which this situation can occur is a hill or pyramid with slopes that are steep at the base, but get flatter towards the top. Intuitively, one would probably select the top of the structure as a scanning position, since it provides the largest viewshed. It covers the entire scene, except the base of the structure: The line of sight to this region is blocked by the structure itself. In order to capture the base of the structure, additional scan positions around the base of the structure are required. However,

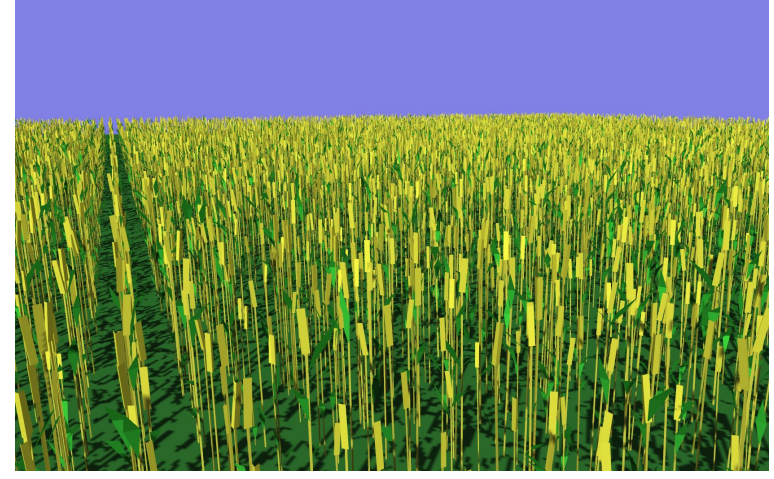

Figure 6: Close-up view of a procedurally generated crop field model.

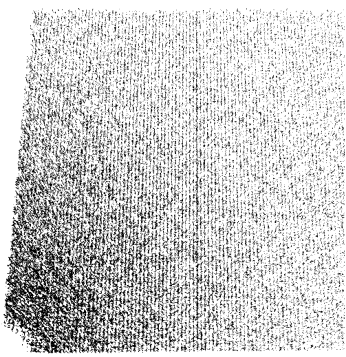

(A)
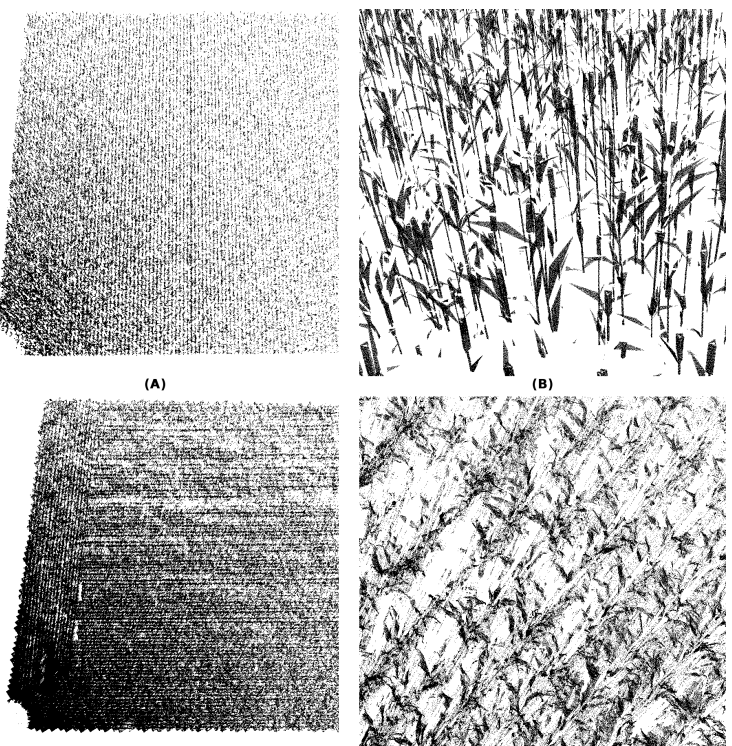

(c)

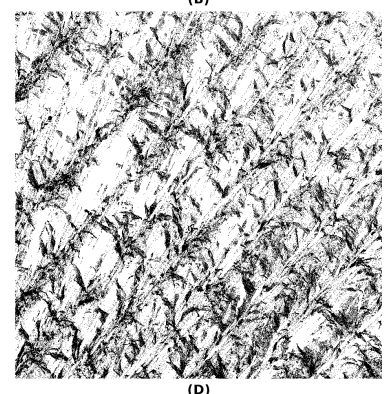

(D)

Figure 7: A procedurally generated crop field point cloud (overview A and close-up B) compared to a real one (overview C and close-up D).

if the combined viewsheds of these additional PSPs cover the entire viewshed of the first PSP on the top of the structure, the latter becomes redundant and can be removed.

A scene with respective geometry was created as a triangle mesh (Fig. 4, and the survey planning algorithm was run on it two times with different removal order criteria. In the first test, redundant PSPs were removed in ascending order of their viewshed size. In the second test, they were removed in ascending order of the arithmetic mean of their line-of-sight test ray incidence angles (black points). The results are shown in figure 5

The result of the first test (white points, removal order based on viewshed size) is suboptimal: The two scan positions at the base of the pyramid structure are placed in positions from where they can not cover the entire scene. Furthermore, their viewing angle on two of the pyramid's sides are close to zero. The second test (black points, removal order based on mean line-of-sight incidence angle) produced a much better result: Very much like a human survey planner would do, it placed two scan positions in front of opposing corners of the pyramid, and none on the top.

\subsection{Example Use Case: Virtual Crop Fields}

One possible use case for HELIOS is the creation of artificial laser scan data to support development of LiDAR point cloud pro- 
cessing and analysis algorithms. One major field of laser scanning research is the investigation of laser scan data from crop fields of different species (e.g. Höfle (2014), Hämmerle and Höfle (2014)). Such data can be used to measure field parameters like plant growth or biomass in precision agriculture scenarios.

In order to show possibilities for the use of HELIOS in this field of research, a "proof of concept" experiment to simulate laser scanning of a virtual crop field was performed. As a first step, a program which can generate $3 \mathrm{D}$ triangle mesh models of crop fields was developed. The program produces crop field models in the Wavefront object (.OBJ) file format, based on a number of parameters like distance between plants in a row, distance between plant rows, plant height, number of leaves, and others. Average values for these parameters are entered by the user, and the program adds random variations to produce more realistic results. A screenshot of a crop field model which was generated this way is shown in figure 6

In the next step, XML scene, platform and scanner definitions were written for a simulated single-position TLS survey of the field. Finally, the simulation was run in HELIOS. Figure 7 shows the result of the simulated scan (Fig. $7 \mathrm{~A}$ and B), and a point cloud from a real crop field scan for comparison (Fig. 7 C C and D). The experiment shows that with the help of additional software to create scene models, HELIOS can be used to produce highly customized virtual laser scan data in a fast and easy way. One great advantage of artificial scan data is that its creation can be controlled very precisely, and that all relevant geometric parameters are immediately available for comparison with the results of data analysis algorithms ("virtual ground truth"). Further experiments have to show to which extent these advantages outweigh the disadvantage of lower realism, compared to performing real scans.

\section{CONCLUSIONS}

Although it is still in an early stage of development, HELIOS can already be used to support a wide variety of laser scanning research projects. The system is designed to integrate all required functionality to evaluate real and imaginary laser scanner hardware and scanning methods, to produce artificial laser scan data and to support TLS survey planning through automatic computation of recommended scanning positions. The main innovation and greatest strength of HELIOS compared to existing LiDAR simulator is its flexibility and modularity. The XML-based "data-driven" simulation engine can quickly be adapted to a wide variety of requirements and simulation scenarios. Also, our realtime $3 \mathrm{D}$ visualization module is a valuable and (to the best of our knowledge) unique improvement over other LiDAR simulators.

The next step is to develop more sophisticated end-user applications that make use of the simulation capabilities of the HELIOS library. One especially interesting possible use case is a graphical front-end that allows direct interaction with a simulated laser scanner, as opposed to the current state of the software which allows only indirect control of the scanner through XML configuration files and the survey playback module. Such an application could be a powerful tool for teaching and practice of laser scanner operations.

\section{ACKNOWLEDGEMENTS}

We thank Martin Hämmerle (Heidelberg University, Germany) and Niko Lukač (University of Maribor, Slovenia) for their valuable contributions to the HELIOS project. We also thank Rainer Hilland for diligent proofreading of this paper.

\section{References}

Bentley, J. L., 1975. Multidimensional binary search trees used for associative searching. Communications of the ACM 18(9), pp. 509-517.

Hämmerle, M. and Höfle, B., 2014. Effects of Reduced Terrestrial LiDAR Point Density on High-Resolution Grain Crop Surface Models in Precision Agriculture. Sensors 14(12), pp. 24212-24230.

Hodge, R. a., 2010. Using simulated Terrestrial Laser Scanning to analyse errors in high-resolution scan data of irregular surfaces. ISPRS Journal of Photogrammetry and Remote Sensing 65(2), pp. 227-240.

Höfle, B., 2014. Radiometric correction of terrestrial LiDAR point cloud data for individual maize plant detection. IEEE Geoscience and Remote Sensing Letters 11(1), pp. 94-98.

Höfle, B., Hollaus, M. and Hagenauer, J., 2012. Urban vegetation detection using radiometrically calibrated small-footprint full-waveform airborne LiDAR data. ISPRS Journal of Photogrammetry and Remote Sensing 67(1), pp. 134-147.

Hollaus, M., Mücke, W., Höfle, B., Dorigo, W., Pfeifer, N., Wagner, W., Bauerhansl, C. and Regner, B., 2009. Tree species classification based on full-waveform airborne laser scanning data. Silvilaser 2009 Proceedings (2008), pp. 54-62.

Kim, S., Min, S., Kim, G., Lee, I. and Jun, C., 2009. Data Simulation of an Airborne LIDAR System. Proceedings of SPIE - The International Society for Optical Engineering 7323(1), pp. 1-10.

Kukko, A. and Hyyppä, J., 2009. Small-footprint Laser Scanning Simulator for System Validation, Error Assessment, and Algorithm Development. Photogrammetric Engineering and Remote Sensing 75(10), pp. 1177-1189.

Lohani, B. and Mishra, R. K., 2007. Generating lidar data in laboratory: Lidar simulator. In: International Archive of Photogrammetry and Remote Sensing XXXVI(3)W52 of Laser Scanning 2007 and SilviLaser 2007, p. 6.

Lovell, J., Jupp, D., Newnham, G., Coops, N. and Culvenor, D., 2005. Simulation study for finding optimal lidar acquisition parameters for forest height retrieval. Forest Ecology and Management 214(1-3), pp. 398-412.

Mallet, C. and Bretar, F., 2009. Full-waveform topographic lidar: State-of-the-art.

Wang, Y. b., Xie, D. b., Yan, G. b., Zhang, W. b. and Mu, X. b. 2013. Analysis on the inversion accuracy of LAI based on simulated point clouds of terrestrial LiDAR of tree by ray tracing algorithm. International Geoscience and Remote Sensing Symposium (IGARSS) pp. 532-535. 